\title{
What Is New in LATEX? V. IATEX on an iPad. Foundation
}

\author{
G. Grätzer
}

I wanted to write an article on how to typeset math on an iPad. It soon became clear that I have to cover a lot of ground on the iPad as a computer before I can discuss LATEX on an iPad. So this "Foundation" lays the groundwork for "Empire", coming soon in the Notices.

\section{Post-PC Revolution}

A few years back, computing was desktop-centric. To update the operating system, for back up, and for many other tasks, you had to connect your smartphone and tablet with a computer.

Tim Cook (Apple's CEO) coined the term "postPC revolution" to describe the trend that a tablet is no longer a younger brother to a PC but an equal partner; in fact, for many users, it may be the only computer they will ever need.

\section{Why the iPad? Let Me Count the Ways}

There are three reasons why the iPad is the only tablet I'll discuss.

1. The iPad's market share is 68 percent. It is clearly the dominant tablet of more than a hundred on the market. (The market share of Android tablets is about 20 percent.)

2 . Today the iPad is the only tablet that is in an ecosystem. (Microsoft's Surface has just joined this exclusive club.) The iPad is just one device under iCloud, along with the iPhone, Mac desktops, and Mac notebooks. I own an iPad, an iPhone, an iMac, and a MacBook Air. A picture I take on my iPhone instantly appears on my iPad. I work on a TEX article on my iMac, and when I am away from home, I continue my work on my MacBook Air-there is no interruption; they are fully synchronized.

G. Grätzer is Distinguished Professor of Mathematics, retired, at the University of Manitoba. His email address is gratzer@me.com.

DOI: http://dx.doi.org/10.1090/noti957

\section{I am not familiar with any other tablet. ${ }^{1}$}

\section{Isn't the iPad Designed for Email, to Read News, and Enjoy Entertainment?}

Certainly. While it has a dual-core CPU, it has a quad-core graphics chip, so viewing videos and complex webpages is quick.

The operating system (iOS 6) is designed to make performing these basic tasks very easy and intuitive. iOS masks the complexities of the underlying computer.

\section{When She Was Good}

To work on an article, Roth sits in front of his computer. In the complex folder hierarchy he finds article.tex, double clicks it to start the IATEX implementation, edits and typesets the article. Then he prints article.pdf, proofreads it, and then goes back to editing...

On the iPad there is only a rectangular array of apps; no documents are visible. There may be folders containing more apps, but no folder in a folder. There are no Library folders, no Download folder. And no File menu containing the Print command! I have article.tex on my desktop, but how do I transfer it to the iPad? Roth plugs in his thumb drive, but it does not work.

In the Mac operating system, there are always features missing, and we can be certain that a future version will incorporate a solution. But this is different; these features are missing on purpose.

Here is what Steve Jobs said about the file system:

"You don't keep your music in the file system, that would be crazy. You keep it in this app that

\footnotetext{
${ }^{1}$ At the TEX User Group conference in Boston this summer, there was a talk about Android pads by Boris Veytsman, "TEX and friends on a pad".
} 
knows about music and knows how to find things in lots of different ways. Same with photos...

"And eventually, the file system management is just gonna be an app for pros, and consumers aren't gonna need to use it."

In this article I will cover the file system and sandboxing, file transfers, and printing. Finally, I will briefly introduce text editing.

\section{File System or Lack Thereof, Sandboxing, File Transfers}

The iPad starts up by displaying a rectangular array of icons and folders for apps. There are no icons for documents.

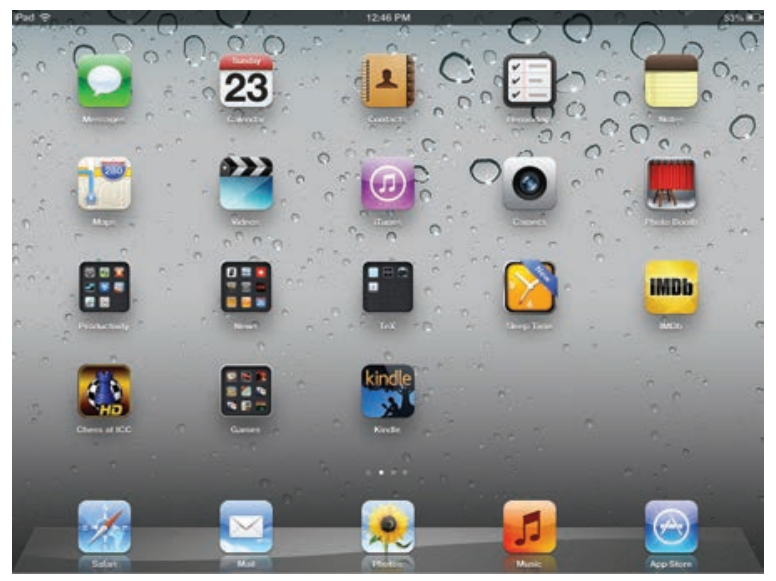

There is no familiar Desktop for documents and folders. No Applications folder. No multiple users. The screen is always occupied by a single window, creating difficulties with Help screens that crowd out the screens they are supposed to help with.

The file system, as we know it from desktop computers, is gone. In its place is an app-centric starting point. Touch the icon of an app and you are in business. When the app opens, you get access to the documents and settings of the app.

For security reasons, the apps are sandboxed, limiting an app's access to files, preferences, network resources, hardware, and so on. Ars Technica's John Siracusa described the goal of sandboxing as follows:

"Running an application inside a sandbox is meant to minimize the damage that could be caused if that application is compromised by a piece of malware. A sandboxed application voluntarily surrenders the ability to do many things that a normal process run by the same user could do. For example, a normal application run by a user has the ability to delete every single file owned by that user. Obviously, a well-behaved application will not do this. But if an application becomes compromised, it may be coerced into doing something destructive."
Of course, the iPad is a computer, and it has a file system; we just do not see it. But it is important to visualize it. To help us along, we will use an app.

\section{FileApp Pro}

If you search the iPad's App Store for "file" apps, there are more than one thousand of them. Many of them could be used to help us understand the iPad file system. I choose FileApp Pro (DigiDNA).

To get started, plug the iPad into a desktop computer, download and start DiskAid on the computer, download and start FileApp Pro on the iPad. On the left panel of DiskAid, click on Apps, then on FileApp. The second pane now shows Imported Files; the right pane shows the files imported to the iPad. In FileApp navigate to Imported Files. Anything you drag into the right pane of DiskAid is copied to FileApp's Imported Files. So much for file transfer.

To see the file structure of the various iPad apps, click on their names. I choose TeX Writer.

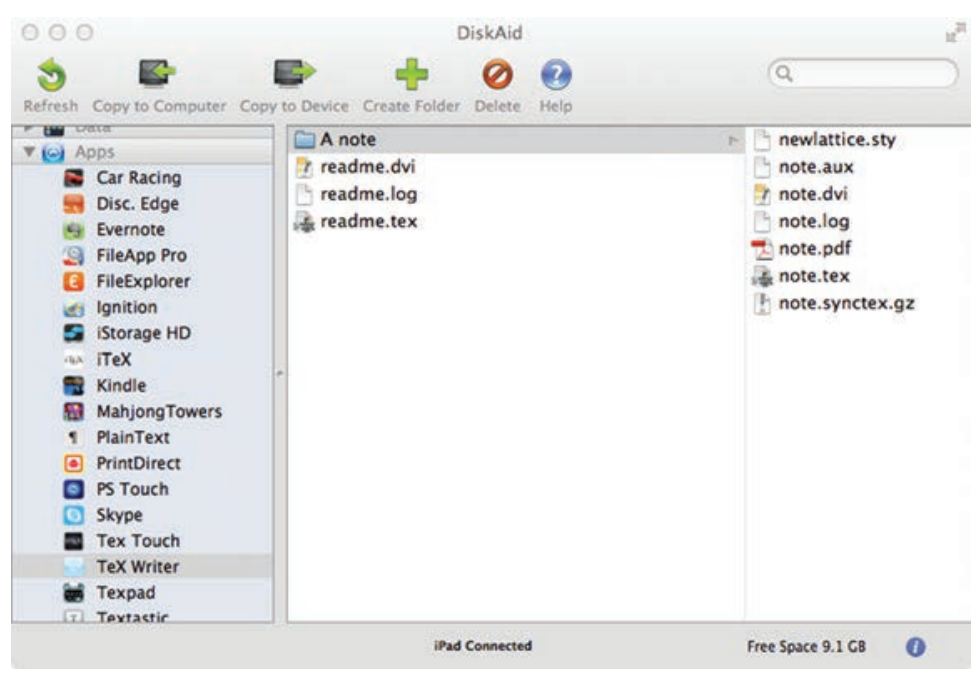

(File App Pro is the Swiss Army knife of utilities. It can zip files, open zip files, create and edit text documents, record sound, and sing lullabies.)

Of course, for file transfers I should also mention the ubiquitous Dropbox. Download it for the iPad, sign in (as you did for your desktop Dropbox), and that's it.

\section{Printing}

When I first wanted to print from my iPhone, I realized that there is no print command; however, lots of apps would do the job. In fact, searching for "print" in the App Store, I discovered over six hundred apps; many of them print, utilizing my desktop computer.

Typical of these apps is PrintDirect (EuroSmartz) and Printer Pro (Readdle Productivity). They can use any printer connected with your desktop computer. 
They wirelessly connect to your computer and print with its help.

If so many apps can help me with printing, why can't iOS? Read the comments to articles about iOS printing; I was not the only one confused.

If the iPad is the poster child of the post-PC revolution, its printing solution cannot involve desktop computers. Apple introduced the appropriate technology; they named it AirPrint. The idea is simple: the iPad collaborates with the printer.

Of course, for this you need a wireless printer that is AirPrint aware. Apple lists all the AirPrintaware printers:

http://support.apple.com/kb/ht4356+ as of this writing, over three hundred.

If you are lucky and have one of these printers, test it. Open an email and touch the Action icon (here it is the Reply icon); this offers you the options Reply, Forward, and Print. Touch Print, Printer Options appears, and you can choose how many copies and on which printer. (Lots of apps provide more choices, such as page range.) Choose the printer and print. For a second test, open a webpage in Safari. There is only one difference: the action icon is a curved arrow in a rectangle.

As a third test, open the Drudge Report. It has the familiar Action icon; we are in business. Finally, open the Politico app, read the news, and look for an Action icon. There is none.

So, to use AirPrint, you need an AirPrint-aware printer and an AirPrint-aware app! For the time being, these are rather severe restrictions.

\section{Text Editors}

Many of us edit LTEX documents in text editors more sophisticated than the text editor that comes with the LTEX implementation. Some thoughts on iPad text editors.

First, writing about apps is like shooting at a moving target. While I was writing about an app, it went through four versions: adding features, removing defective ones. This is especially true of the LATEX implementations I will write about in the next article. Some have no documentation. One has a single page, explaining why it can only do TEX, not LTEX. In fact, it can do LTEX.

Second, there are so many text editors, well over two hundred. Take a look at the table at http://brettterpstra.com/ios-text-editors/ This table is a $50 \times 31$ matrix, each row representing a text editor, each column representing a feature (such as Search and Replace). The entries are Yes or No. Hovering over the name of a text editor, you get a listing of additional features and the App Store information.

Third, keeping the iPad horizontal, the keyboard gobbles up too much real estate. Keeping it vertical, the keyboard is less intrusive, but the keys are smaller. If you want to do serious work on the iPad, buy a keyboard.

Fourth, the iOS's touch text editing is nice, but it lacks a feature crucial for text editing: moving the cursor a character ahead or back. (Of course, Bluetooth keyboards have cursor keys!) Text editors offer a variety of solutions, for instance, finger swiping.

I will discuss briefly a very sophisticated text editor: Textastics. If you want Syntax Highlighting, Search and Replace, and Text Expander, this is your only choice.

Here you see me editing this article:

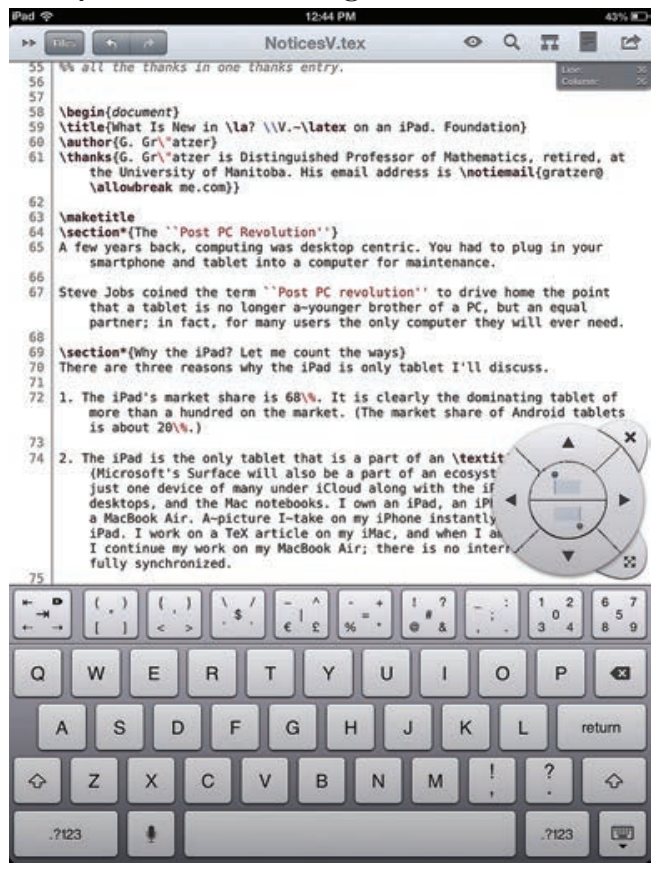

You can see the extra keyboard row and the cursor navigation wheel (which appears with a two finger tap; finger swipe also moves the cursor). It comes with an excellent user manual. (Textastics can also perform a number of nonediting tasks, such as zipping and unzipping files.)

Textastics has no Mac version, and if you spend time shaping it to your liking, then you would like the same tamed editor for all your work. The good news is, it's coming. I can hardly wait to see it.

\section{Onward}

I hope I have convinced you that the iPad is a computer. So what we need now are some LATEX implementations on the iPad computer.

\section{Acknowledgments}

Drafts of this article were read and commented on by a number of experts. I am grateful, in particular, to Alexander Blach, Richard Koch, Scot Mcphee, and Jonathan Weisberg. 